\title{
Association between Nephrotoxic Drug Combinations and Acute Kidney Injury in the Neonatal Intensive Care Unit
}

\author{
Sara N. Salerno, PharmD ${ }^{1, \star}$, Yuting Liao, PharmD ${ }^{2, \star}$, Wesley Jackson, MD, MPH ${ }^{3}$, Rachel G. Greenberg, MD, MB, MHS ${ }^{4,5}$, \\ Cameron J. McKinzie, PharmD², Ashley McCallister, PharmD, MBA ${ }^{2}$, Daniel K. Benjamin, $\mathrm{PhD}^{6}$, Matthew M. Laughon, MD, \\ $\mathrm{MPH}^{3}$, Keia Sanderson, MD, MSCR${ }^{7}$, Reese H. Clark, MD ${ }^{8}$, and Daniel Gonzalez, PharmD, $\mathrm{PhD}^{1}$
}

Objective To determine the incidence of acute kidney injury (AKI) in infants exposed to nephrotoxic drug combinations admitted to 268 neonatal intensive care units managed by the Pediatrix Medical Group.

Study design We included infants born at 22-36 weeks gestational age, $\leq 120$ days postnatal age, exposed to nephrotoxic drug combinations, with serum creatinine measurements available, and discharged between 2007 and 2016. To identify risk factors associated with a serum creatinine definition of AKI based on the Kidney Disease: Improving Global Outcomes criteria, we performed multivariable logistic and Cox regression adjusting for gestational age, sex, birth weight, postnatal age, race/ethnicity, sepsis, respiratory distress syndrome, baseline serum creatinine, and duration of combination drug exposure. The adjusted odds of AKI were determined relative to gentamicin + indomethacin for the following nephrotoxic drug combinations: chlorothiazide + ibuprofen; chlorothiazide + indomethacin; furosemide + gentamicin; furosemide + ibuprofen; furosemide + tobramycin; ibuprofen + spironolactone; and vancomycin + piperacillin-tazobactam.

Results Among 8286 included infants, 1384 (17\%) experienced AKI. On multivariable analysis, sepsis, lower baseline creatinine, and duration of combination therapy were associated with increased odds of AKI. Furosemide + tobramycin and vancomycin + piperacillin-tazobactam were associated with a decreased risk of AKI relative to gentamicin + indomethacin in both the multivariable and Cox regression models.

Conclusions In this cohort, infants receiving longer durations of nephrotoxic combination therapy had an increased odds of developing AKI. (J Pediatr 2021;228:213-9).

cute kidney injury (AKI) in critically ill infants is common and associated with poor outcomes and high mortality. ${ }^{1}$ The Assessment of Worldwide Acute Kidney Epidemiology in Neonates study performed in critically ill neonates across 24 neonatal intensive care units (NICUs) reported a 30\% incidence of AKI overall and $18 \%, 48 \%$, and 37\% for gestational ages of 22-28 weeks, 29-35 weeks, and $\geq 36$ weeks, respectively. In the Assessment of Worldwide Acute Kidney Epidemiology in Neonates study, infants with AKI had higher mortality $(9.7 \%$ vs $1.4 \% ; P<.001)$ and longer hospital stays compared with infants without AKI after adjusting for known risk factors. ${ }^{2}$ Infant risk factors that have been associated with AKI from small single-site studies include very low birth weight (VLBW), congenital heart disease, perinatal asphyxia, hypoxic ischemic encephalopathy, sepsis, respiratory distress, and extracorporeal membrane oxygenation. $^{3}$

Besides these identified risk factors, nephrotoxic drugs are commonly administered to infants and have also been associated with an increased risk of AKI among critically ill infants. ${ }^{6-8}$ The most common nephrotoxic drugs administered to infants in the NICU include gentamicin, vancomycin, furosemide, indomethacin, tobramycin, acyclovir, chlorothiazide, spironolactone, piperacillin-tazobactam, amphotericin B, and ibuprofen. ${ }^{9}$ A study in 1828 critically ill children, including term infants $\geq 2$ weeks of age, reported an increased risk for AKI as the number of nephrotoxic drugs increased (OR, 1.3; 95\% CI, 1.2-1.4). ${ }^{8}$ Furthermore, in a sample of 107 VLBW infants in the NICU, infants who experienced AKI received more nephrotoxic drugs per day than those who did not experience AKI ( 0.24 vs $0.15 ; P=.003)$.

A single-site study of 86 term newborns in the NICU reported that infants are at high risk of AKI when treated with aminoglycosides in combination with diuretics for $>4.5$ days. ${ }^{10}$ Similarly, nonsteroidal anti-inflammatory drugs

\footnotetext{
AKI Acute kidney injury

NICU Neonatal intensive care unit

VLBW Very low birth weight
}

From the ${ }^{1}$ Division of Pharmacotherapy and

Experimental Therapeutics, UNC Eshelman School of Pharmacy, The University of North Carolina at Chapel Hill; ${ }^{2}$ Department of Pharmacy, University of North Carolina Medical Center, Chapel Hill; ${ }^{3}$ Department of Pediatrics, School of Medicine, The University of North Carolina at Chapel Hill, Chapel Hill; ${ }^{4}$ Department of

Pediatrics, Duke University School of Medicine, Durham ${ }^{5}$ Duke Clinical Research Institute, Durham, NC; ${ }^{6}$ Department of Economics, Clemson University, Clemson, SC; ${ }^{7}$ Department of Medicine-Nephrology, UNC School of Medicine, The University of North Carolina at Chapel Hill, Chapel Hill, NC; and the

${ }^{8}$ MEDNAX Center for Research, Education, Quality and Safety, Sunrise, FL

${ }^{*}$ Contributed equally.

S.S. was supported by the National Institute of Genera Medical Sciences and the Eunice Kennedy Shriver National Institute of Child Health and Human Development of the National Institutes of Health under Award Number TT2 the National Heart, Lung, and Blood Institute (1K24HL143283). D.G. received research support from the Eunice Kennedy Shriver National Institute of Child Health and Human Development (5K23HD083465). The content is solely the responsibility of the authors and does not necessarily represent the official views of the chation from in lnstitutes of Healh. R.G. has received support from industry for research services (https.//dcri.org/ about-us/conflict-of-interest/). The other authors declare no conflicts of interest. 
increased the risk of $\mathrm{AKI}$ in neonates receiving vancomycin with and without gentamicin with an OR of 5.2 $(P<.001) .^{11}$ Furthermore, 10 preterm infants with patent ductus arteriosus who received gentamicin + indomethacin were reported to have significant elevations in gentamicin concentrations and serum creatinine and decreases in 24hour urine output and fluid intake after coadministration with indomethacin. ${ }^{12}$ The primary aim of this study was to use a large multicenter database of infants to determine the incidence of AKI and the relative nephrotoxicity of nephrotoxic drug combinations likely encountered in the NICU.

\section{Methods}

We identified infants $\leq 120$ days postnatal age (those hospitalized for longer times are likely to have other comorbidities), born at 22-36 weeks gestational age, exposed to only 1 nephrotoxic drug combination, discharged from any of 268 NICUs managed by the Pediatrix Medical Group between 2007 and 2016, and with serum creatinine values available in the medical chart. The Pediatrix Medical Group maintains electronic medical data prospectively captured through notes generated by clinicians including: admission history and physicals, daily progress notes, laboratory results, discharge summaries, drugs, diagnoses, and maternal history and demographics. We excluded infants that were predisposed to AKI to decrease confounding of the association between AKI and nephrotoxic drugs. The following exclusion criteria were applied: infants with hypoxic ischemic encephalopathy, those with underlying congenital renal dysfunction or disease, and those who received dialysis before receiving nephrotoxic drugs, or received extracorporeal membrane oxygenation. The study was approved by the Duke University Institutional Review Board with a waiver of informed consent.

\section{Definitions}

The association between AKI and nephrotoxic drug exposure was evaluated for the following drug combinations: chlorothiazide + ibuprofen; chlorothiazide + indomethacin; furosemide + gentamicin; furosemide + ibuprofen; furosemide + tobramycin; ibuprofen + spironolactone; vancomycin + piperacillin-tazobactam; and gentamicin + indomethacin. These combinations were selected by entering into Micromedex the top 50 drugs commonly administered to infants discharged from NICUs managed by the Pediatrix Medical Group, and then filtering by those drug combinations based on the classification system within Micromedex in which there was good or excellent evidence for a nephrotoxic drug-drug interaction in either adults or pediatric patients. Excellent evidence was defined by Micromedex as the existence of controlled studies clearly establishing an interaction. Good evidence was defined by Micromedex as the existence of documentation strongly suggesting an interaction but lacking well-controlled studies (Figure). We excluded patients who received $>1$ of these drug combinations during hospitalization. For infants receiving multiple courses of the same drug combination, we included serum creatinine data for the first course only.

Based on the Kidney Disease: Improving Global Outcomes 2012 guidelines, we defined AKI as an increase in serum creatinine $\geq 0.3 \mathrm{mg} / \mathrm{dL}$ or $\geq 1.5$ times the baseline creatinine, which was considered to be the most recent serum creatinine value within 2 days of first drug combination exposure. ${ }^{13} \mathrm{We}$ excluded all serum creatinine values of $>10 \mathrm{mg} / \mathrm{dL}$ owing to our concern that these values may have been entered in error. Serum creatinine values during the first 3 postnatal days were excluded because these levels are more reflective of maternal serum creatinine values. ${ }^{14,15}$ We used a serum creatininebased AKI definition because the Pediatrix database did not capture urine output. Drug combination exposure was defined as the administration of both drugs to the same infant on the same day. AKI was attributed to the nephrotoxic drug combination if it occurred after the first day of exposure through 6 days after last day of drug combination exposure. Beyond 6 days, concentrations for all drugs evaluated were

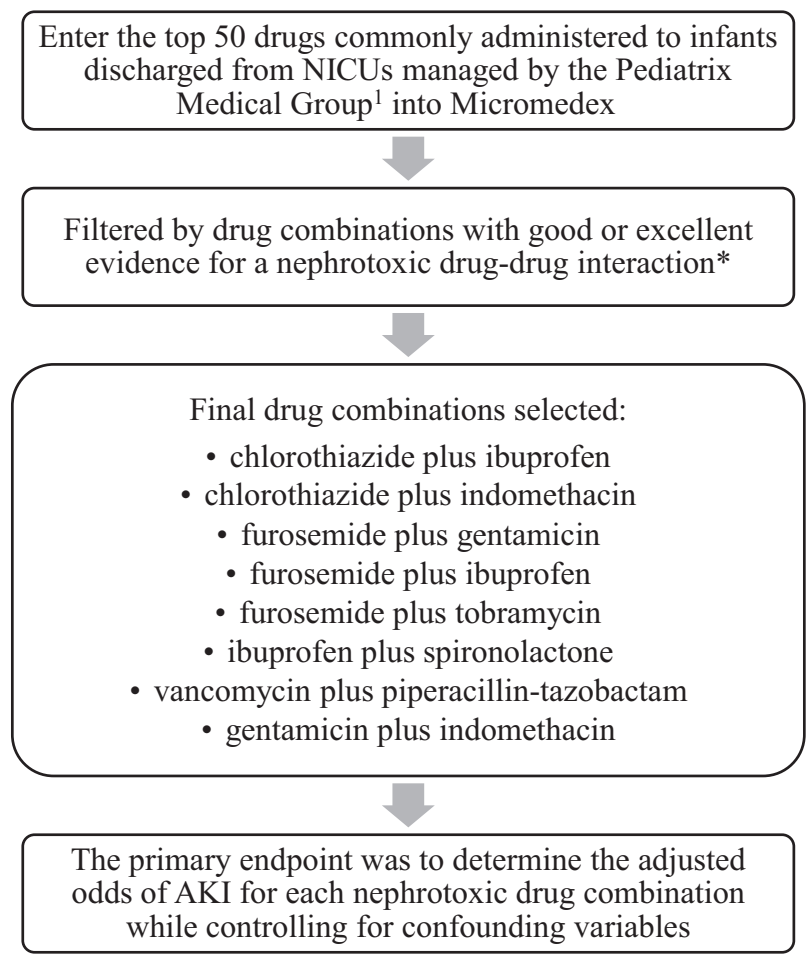

Figure. Approach for determining drug combinations associated with $\mathrm{AKI}$ risk in infants. * Excellent evidence was defined as the existence of controlled studies clearly establishing an interaction. Good evidence was defined as the existence of documentation strongly suggesting an interaction but lacking well-controlled studies. ${ }^{1} \mathrm{H}$ sieh EM, Hornik CP, Clark RH, Laughon MM, Benjamin DK Jr, Smith PB; Best Pharmaceuticals for Children Act-Pediatric Trials Network. Medication use in the neonatal intensive care unit. Am J Perinatol 2014;31:811-21. 
considered to be negligible based on the passage of 5 half-lives of the drugs. ${ }^{16}$ For example, the drug evaluated with the longest half-life, furosemide, has an average half-life in neonates ranging from 7.7 to 26.8 hours. ${ }^{17}$ Although we only considered the first course of combination therapy, if the same drug combination was stopped and resumed between the first day and 6 days after the last day of drug exposure, then this was counted as 1 course of drug combination exposure. Demographic data included gestational age, sex, birth weight, race, and postnatal age. Sepsis was defined as the presence of any positive culture. Respiratory distress syndrome was defined as the exposure to surfactant in the first 7 postnatal days.

\section{Statistical Analyses}

We used the $\chi^{2}$ test to examine demographic differences between infants receiving nephrotoxic drug combinations who experienced AKI and infants receiving nephrotoxic drug combinations who did not experience AKI. Categorical variables were presented as count (percentages) and continuous variables were presented as median (IQR). Gestational age was stratified by <24, 24-26, 27-29, 30-32, and 33-36 weeks; birth weight was stratified by $\leq 750,751-1000,1001-1500$, $1501-2500$, and $>2500 \mathrm{~g}$; and postnatal age was stratified by $<2,2-3,4-5,6-7$, and 8-16 weeks.

To estimate the association between nephrotoxic drug combinations and AKI, we used both a logistic model with random effects across sites and a Cox survival model with shared frailty across sites, the latter allowing for multiple failures (new instances of AKI) for each patient. With the logistic model, the outcome was AKI at any time under observation, and drug exposure was the total number of days the patient was treated with the drug combination. For the Cox model, drug exposure relevant to each day was the number of the prior 6 days on which the patient was treated with the drug combination (thus allowing for nephrotoxic drug to clear from the body, as previously described). Both models controlled for gestational age, sex, birth weight, postnatal age, race, sepsis, respiratory distress syndrome, and baseline serum creatinine. Significance for all tests was set at $P<.05$. Stata version 16.1 (StataCorp, College Station, Texas) was used to perform the statistical analysis.

\section{Results}

\section{Clinical Characteristics}

We identified 23399 infants exposed to nephrotoxic drug combinations who were admitted to the Pediatrix Medical Group affiliated NICUs between 2007 and 2016. Of the 23 399 patients meeting inclusion criteria, 15113 were excluded because they did not have $\geq 2$ serum creatinine measurements available, resulting in 8286 infants for analysis. We excluded $<1 \%$ of serum creatinine values $>10 \mathrm{mg} / \mathrm{dL}$ owing to our concern that these values may have been entered in error. There were an average of 6 serum creatinine measurements per infant in the group that experienced AKI and an average of 5 serum creatinine measurements per infant in the group that did not experience AKI. The overall incidence of AKI was $17 \%(1384 / 8286)$ in infants exposed to nephrotoxic drug combinations ( $11 \%$ were diagnosed as stage $1,4 \%$ were diagnosed as stage 2 , and $2 \%$ were diagnosed as stage 3 ). The median birth weight was $920 \mathrm{~g}$ (IQR, 700-1400 g) in infants with AKI and $945 \mathrm{~g}$ (IQR, 710-1395 g) in infants without AKI. The median gestational age was 27 weeks (IQR, 25-31 weeks) in infants with AKI and was 27 weeks (IQR, 25-30 weeks) in infants without AKI. The median postnatal age at start of drug combination was 10 weeks (IQR, 5-25 weeks) for infants with AKI and 11 weeks (IQR, 5-26 weeks) for infants without AKI. The median duration of combination therapy was 3 days (IQR, 1-6 days) for infants with AKI and was 2 days (IQR, 1-5 days) for infants without AKI. Infant demographics that differed between infants who experienced AKI and who did not experience AKI included postnatal age, race, sepsis, and length of combination therapy (Table I).

\section{Risk Factors for AKI}

In the logistic model, sepsis, lower baseline creatinine, and longer duration of combination therapy were associated with an increased risk of AKI (Table II). There were no differences between infants with and without AKI across birth weight, postnatal or gestational age groups, sex, race, or respiratory distress syndrome status (Table II). In the Cox model, longer duration of combination therapy was associated with increased risk of AKI, as were birth weight $<1000$ g, postnatal age $<2$ weeks, Hispanic race, lower baseline creatinine, and sepsis. Gestational age between 27 and 29 weeks and respiratory distress syndrome were associated with a decreased risk of AKI (Table III).

\section{Nephrotoxic Drug Combinations Associated with AKI}

No infants received chlorothiazide + ibuprofen or ibuprofen + spironolactone. The prevalence of AKI was 316 of 1788 (18\%) for gentamicin + indomethacin, 2 of 6 (33\%) for chlorothiazide + indomethacin, 697 of 4265 (16\%) for furosemide + gentamicin, 3 of 25 (12\%) for furosemide + ibuprofen, 94 of 636 (15\%) for furosemide + tobramycin, and 272 of 1566 (17\%) for vancomycin + piperacillin-tazobactam. The median postnatal age for the initiation of nephrotoxic drug combinations was 23 days (IQR, 22-46) for chlorothiazide + indomethacin; 9 days (IQR, 2-29 days) for furosemide + gentamicin; 12 days (IQR, 6-18 days) for furosemide + ibuprofen; 28 days (IQR, 16-47 days) for furosemide + tobramycin; 1 day (IQR, 0-3 days) for gentamicin + indomethacin; and 15 days (IQR, 8-29 days) for vancomycin + piperacillin-tazobactam.

Furosemide + tobramycin and vancomycin + piperacillintazobactam were associated with a decreased risk of AKI in both the logistic model and the Cox model, relative to the reference group (gentamicin + indomethacin) (Table II and Table III). Chlorothiazide + indomethacin was associated with an increased risk of AKI relative to gentamicin + indomethacin in the Cox (but not logistic) model, but the sparse usage of chlorothiazide + indomethacin $(\mathrm{n}=6)$ 
Table I. Demographics and clinical characteristics of infants born at 22-36 weeks gestation between 2007 and 2016

\begin{tabular}{|c|c|c|c|}
\hline $\begin{array}{l}\text { Demographics or } \\
\text { clinical characteristics }\end{array}$ & $\begin{array}{l}\text { Experienced AKI } \\
(\mathrm{n}=1384[17 \%])\end{array}$ & $\begin{array}{l}\text { Did not experience AKI } \\
\quad(\mathrm{n}=6902[83 \%])\end{array}$ & $\begin{array}{l}P \\
\text { value }\end{array}$ \\
\hline $\begin{array}{l}\text { Length of combination } \\
\text { therapy, median (IQR) }\end{array}$ & $3(1-6)$ & $2(1-5)$ & $<.01$ \\
\hline Birth weight $(\mathrm{g})$ & & & .07 \\
\hline$\leq 750$ & $438(32)$ & $2017(29)$ & \\
\hline $751-1000$ & $340(25)$ & $1698(25)$ & \\
\hline $1001-1500$ & $287(21)$ & 1663 (24) & \\
\hline $1501-2500$ & $226(16)$ & $1100(16)$ & \\
\hline$>2500$ & $93(7)$ & $424(6)$ & \\
\hline Gestational age (weeks) & & & .20 \\
\hline$<24$ & $97(7)$ & $433(6)$ & \\
\hline $24-26$ & $506(37)$ & $2478(36)$ & \\
\hline $27-29$ & $360(26)$ & $1917(28)$ & \\
\hline $30-32$ & 180 (13) & $991(14)$ & \\
\hline $33-36$ & $241(17)$ & $1083(16)$ & \\
\hline Postnatal age (weeks) & & & .01 \\
\hline$<2$ & $850(62)$ & $3977(58)$ & \\
\hline $2-3$ & $248(18)$ & $1441(21)$ & \\
\hline $4-5$ & $122(9)$ & 742 (11) & \\
\hline $6-7$ & $71(5)$ & $349(5)$ & \\
\hline $8-16$ & $85(6)$ & $376(5)$ & \\
\hline Male sex & $789(57)$ & 3927 (57) & .92 \\
\hline Race/ethnicity & & & .01 \\
\hline White & $523(39)$ & $2735(41)$ & \\
\hline Black & $306(23)$ & $1631(25)$ & \\
\hline Hispanic & $448(34)$ & $1938(29)$ & \\
\hline Other & $57(4)$ & $344(5)$ & \\
\hline Sepsis & $805(58)$ & $3753(54)$ & .01 \\
\hline $\begin{array}{l}\text { Respiratory distress } \\
\text { syndrome }\end{array}$ & 995 (72) & 4948 (72) & .88 \\
\hline \multicolumn{4}{|c|}{$\begin{array}{l}\chi^{2} \text { tests were used to examine demographic differences between infants receiving nephrotoxi } \\
\text { drug combinations who experienced AKI and infants receiving nephrotoxic drug combination } \\
\text { who did not experience AKI. For some of the covariates, there were missing data recorded in the } \\
\text { database ( } 25 \text { for postnatal age, } 304 \text { for race/ethnicity, and } 8 \text { for sex). Categorical variable } \\
\text { were presented as count (column percentages) relative to the total number of subjects witl } \\
\text { and without AKI, respectively. Significance was established at a } P \text { value of }<.05 \text {. }\end{array}$} \\
\hline
\end{tabular}

makes us reluctant to put much weight on this finding (Table III).

\section{Discussion}

Although nephrotoxic drug exposure has been associated with AKI in VLBW infants and critically ill term infants $\geq 2$ weeks of age, this multicenter study investigated AKI after exposure to combinations of nephrotoxic drugs in infants in the NICU of varying gestational ages and birth weights. ${ }^{6-}$ ${ }^{8} \mathrm{We}$ found that infants receiving longer nephrotoxic combination therapy were more likely to develop AKI. A study of VLBW infants also reported that infants who experienced AKI had greater total nephrotoxic drug days than infants who did not experience AKI (23.9 days vs 9.9 days; $P<.0001){ }^{7}$ Similarly, in hospitalized noncritically ill patients aged 1 day to 18 years of age, patients with AKI were exposed to more nephrotoxic drugs. In the Assessment of Worldwide Acute Kidney Epidemiology in Neonates study, the incidence of AKI was highest in the oldest ( $>36$ weeks) and youngest ( $\geq 22$ to $<29$ weeks) gestational age groups. ${ }^{2}$ It is likely that younger gestational age is associated with AKI, but in our sample, only the 27-29 week group with the Cox model
Table II. Random effects logistic model of AKI among infants born at 22-36 weeks gestation between 2007 and 2016

\begin{tabular}{|c|c|c|}
\hline Categories & AKI OR (95\% Cl) & $P$ value \\
\hline \multicolumn{3}{|l|}{ Nephrotoxic drug combination } \\
\hline Chlorothiazide + indomethacin & $2.95(0.50-17.5)$ & .23 \\
\hline Furosemide + gentamicin & $0.94(0.79-1.13)$ & .51 \\
\hline Furosemide + ibuprofen & $0.76(0.22-2.64)$ & .67 \\
\hline Furosemide + tobramycin & $0.70(0.52-0.95)$ & .02 \\
\hline $\begin{array}{l}\text { Vancomycin + piperacillin- } \\
\text { tazobactam }\end{array}$ & $0.77(0.61-0.98)$ & .03 \\
\hline Gentamicin + indomethacin & Reference & \\
\hline Duration of therapy (days) & $1.04(1.02-1.06)$ & $<.01$ \\
\hline Baseline creatinine & $0.62(0.50-0.78)$ & $<.01$ \\
\hline \multicolumn{3}{|l|}{ Birth weight (g) } \\
\hline$\leq 750$ & $1.35(0.86-2.13)$ & .19 \\
\hline $751-1000$ & $1.20(0.78-1.86)$ & .40 \\
\hline $1001-1500$ & $1.02(0.69-1.52)$ & .92 \\
\hline $1501-2500$ & $1.01(0.75-1.37)$ & .93 \\
\hline$>2500$ & Reference & \\
\hline \multicolumn{3}{|l|}{ Gestational age (weeks) } \\
\hline$<24$ & $0.92(0.58-1.46)$ & .72 \\
\hline $24-26$ & $0.85(0.58-1.26)$ & .42 \\
\hline $27-29$ & $0.86(0.60-1.21)$ & .38 \\
\hline $30-32$ & $0.86(0.65-1.15)$ & .31 \\
\hline $33-36$ & Reference & \\
\hline \multicolumn{3}{|l|}{ Postnatal age (weeks) } \\
\hline$<2$ & $1.33(0.98-1.80)$ & .07 \\
\hline $2-3$ & $0.98(0.73-1.33)$ & .91 \\
\hline $4-5$ & $0.81(0.58-1.12)$ & .20 \\
\hline $6-7$ & $1.05(0.72-1.51)$ & .81 \\
\hline $8-16$ & Reference & \\
\hline Male & $1.03(0.91-1.17)$ & .62 \\
\hline \multicolumn{3}{|l|}{ Race/ethnicity } \\
\hline Black & $0.92(0.78-1.10)$ & .37 \\
\hline Hispanic & $1.11(0.94-1.31)$ & .23 \\
\hline Other & $0.82(0.60-1.12)$ & .22 \\
\hline White & Reference & \\
\hline Sepsis & $1.25(1.09-1.44)$ & $<.01$ \\
\hline Respiratory distress syndrome & $0.96(0.82-1.12)$ & .59 \\
\hline
\end{tabular}

Multivariable logistic regression was performed to determine the association between AKI and nephrotoxic drug combinations (relative to the arbitrarily chosen reference group, gentamicin + indomethacin) adjusting for the following confounding variables: gestational age, sex, birth weight, postnatal age, race, sepsis, respiratory distress syndrome, and duration of combination drug exposure. Random effects by site were assumed, to control for differences across sites. Of the 8286 infants meeting inclusion criteria, 7913 were included in this regression analysis because Stata SE excludes subjects missing values for any of these covariates. Baseline creatinine was defined as the most recent serum creatinine value within 2 days of first drug combination exposure. Significance was established at $P<.05$. None of the infants received chlorothiazide + ibuprofen or ibuprofen + spironolactone.

reached statistical significance for a lower risk of AKI relative to the 33-35 week group. Similar to previous studies in infants, sepsis was positively associated with AKI in this cohort of infants. ${ }^{4,18-20}$ A postnatal age of $<2$ weeks was associated with AKI in the Cox model estimates. Infants are particularly susceptible to AKI soon after birth as the postnatal kidney adapts to the extrauterine environment. The glomerular filtration rate increases from about 5 to $40 \mathrm{~mL} / \mathrm{min} /$ $1.73 \mathrm{~m}^{2}$ during the first week of life owing to increasing central to peripheral renal blood flow and decreasing renal vascular resistance. ${ }^{1}$ Thus, glomeruli, which reside in the more peripheral renal cortex, are particularly more susceptible to insult as a result. In addition, a birth weight of $<1000 \mathrm{~g}$ was associated in the Cox model with an increased risk of AKI relative to infants $>2500 \mathrm{~g}$. A study in VLBW infants reported that there was an inverse linear relationship between birth 
Table III. Shared frailty Cox proportional hazards model of AKI in preterm infants (born at 22-37 weeks of gestation) between 2007 and 2016

\begin{tabular}{|c|c|c|}
\hline Category & $\begin{array}{l}\text { Hazard Ratio } \\
\text { (HR) }(95 \% \mathrm{CI})\end{array}$ & $P$ value \\
\hline \multicolumn{3}{|l|}{ Nephrotoxic drug combination } \\
\hline Chlorothiazide + indomethacin & $2.73(1.04-7.16)$ & \\
\hline Furosemide + gentamicin & $1.01(0.90-1.13)$ & \\
\hline Furosemide + ibuprofen & $0.44(0.14-1.37)$ & \\
\hline Furosemide + tobramycin & $0.72(0.58-0.88)$ & $<.01$ \\
\hline $\begin{array}{l}\text { Vancomycin + piperacillin- } \\
\text { tazobactam }\end{array}$ & $0.81(0.70-0.94)$ & $<.01$ \\
\hline Gentamicin + indomethacin & Reference & \\
\hline Duration of therapy (days) & $1.06(1.02-1.10)$ & $<.01$ \\
\hline Baseline creatinine & $0.54(0.46-0.62)$ & $<.01$ \\
\hline \multicolumn{3}{|l|}{ Birth weight $(\mathrm{g})$} \\
\hline$\leq 750$ & $1.60(1.20-2.11)$ & $<.01$ \\
\hline $751-1000$ & $1.44(1.10-1.88)$ & $<.01$ \\
\hline $1001-1500$ & $1.18(0.92-1.51)$ & \\
\hline $1501-2500$ & $1.14(0.94-1.38)$ & \\
\hline$>2500$ & Reference & \\
\hline \multicolumn{3}{|l|}{ Gestational age (weeks) } \\
\hline$<24$ & $0.76(0.56-1.02)$ & \\
\hline $24-26$ & $0.85(0.67-1.08)$ & \\
\hline $27-29$ & $0.77(0.62-0.96)$ & \\
\hline $30-32$ & $0.85(0.72-1.02)$ & \\
\hline $33-35$ & Reference & \\
\hline \multicolumn{3}{|l|}{ Postnatal age (weeks) } \\
\hline$<2$ & $1.44(1.19-1.75)$ & $<.01$ \\
\hline $2-3$ & $1.11(0.92-1.34)$ & \\
\hline $4-5$ & $0.83(0.68-1.02)$ & \\
\hline $6-7$ & $1.02(0.81-1.28)$ & \\
\hline $8-16$ & Reference & \\
\hline Male sex & $1.06(0.98-1.15)$ & \\
\hline \multicolumn{3}{|l|}{ Race/ethnicity } \\
\hline Black & $0.99(0.88-1.11)$ & \\
\hline Hispanic & $1.13(1.01-1.26)$ & \\
\hline Other & $0.98(0.81-1.20)$ & \\
\hline White & Reference & \\
\hline Sepsis & $1.13(1.04-1.23)$ & $<.01$ \\
\hline Respiratory distress syndrome & $0.89(0.81-0.98)$ & \\
\hline
\end{tabular}

A Cox proportional hazards model was estimated to determine the association between AKI and nephrotoxic drug combinations (relative to the arbitrarily chosen reference group, gentamicin + indomethacin) adjusting for the time-dependent variable (duration of combination therapy) and other fixed covariates (gestational age, sex, birth weight, postnatal age, race, sepsis, and respiratory distress syndrome). Shared frailty within sites was assumed to control for site specific effects. Significance was established at $P<.05$. Baseline creatinine was defined as the most recent serum creatinine value within 2 days of first drug combination exposure. None of the infants received chlorothiazide + ibuprofen or ibuprofen + spironolactone.

weight and nephrotoxic drugs received per day which increased their risk of AKI. ${ }^{7}$ A lower baseline serum creatinine was associated with an increased risk of AKI, which is likely because preterm neonates can have low serum creatinine levels and small increases can lead to a classification of AKI using our serum creatinine based AKI definition.

The overall incidence of AKI in this cohort of infants exposed to nephrotoxic drug combinations was $17 \%$. The incidence of AKI in infants is highly variable depending on the definition of AKI used, the population studied, and the number of study sites, infants, and serum creatinine measurements available. ${ }^{1,2,21,22}$ For example, a single-site study of 312 infants reported that $24 \%$ of infants developed AKI based on a decrease in the glomerular filtration rate of $\geq 25 \%$ from baseline or a decrease in urine output of $<1.5 \mathrm{~mL} / \mathrm{kg} /$ hour for 24 hours. ${ }^{23}$ Another single-site study of 1992 neonates reported an AKI prevalence of $8.4 \%$ based on an increase in the serum creatinine of $>1.5 \mathrm{mg} / \mathrm{dL}$ for $>24$ hours or that increased at the rate of $0.3 \mathrm{mg} / \mathrm{dL} /$ day after the first 48 hours of birth. ${ }^{24}$ Moreover, a recently published multicenter cohort study in 2022 infants identified a 30\% incidence of AKI using the following AKI definition: serum creatinine increase of $\geq 0.3 \mathrm{mg} / \mathrm{dL}$ or $\geq 50 \%$ higher than previous lowest value, or if urine output was $<1 \mathrm{~mL} / \mathrm{kg} /$ hour on postnatal days $2-7 .^{2}$

Furosemide + tobramycin and vancomycin + piperacillintazobactam were associated with a decreased risk of AKI relative to gentamicin + indomethacin. A study of 10 preterm infants with patent ductus arteriosus who received gentamicin + indomethacin reported a significant elevation in gentamicin peak and trough concentrations and a slight but significant increase in serum creatinine before and after coadministration with indomethacin. ${ }^{12}$ Indomethacin can decrease glomerular filtration by inhibiting renal prostaglandin production, which may result in decreased clearance of gentamicin, leading to gentamicin accumulation and nephrotoxicity. ${ }^{12,25,26}$ Several studies have reported that tobramycin is less nephrotoxic than gentamicin in adult patients. Tobramycin was significantly less nephrotoxic than gentamicin in a study performed in 201 critically ill older adults $(37 \%$ vs $22 \% ; P<.02)$ using a serum creatinine definition of AKI. ${ }^{27}$ Additionally, a prospective, randomized, double-blind trial in 258 adult patients with suspected sepsis treated with tobramycin or gentamicin also reported that nephrotoxicity occurred less frequently with tobramycin than gentamicin $(26 \%$ vs $12 \% ; P<.025) .{ }^{28}$ Another prospective study in 62 adult patients reported that gentamicin was associated with renal failure more than three times more often than tobramycin. ${ }^{29}$ Several studies have suggested that nephrotoxicity for vancomycin administered alone or in combination with gentamicin in premature infants and pediatric patients is extremely rare. ${ }^{30-32}$ However, a retrospective study in pediatric patients (median, 0.22 years; $\mathrm{IQR}, 0-6.3$ years) receiving piperacillin-tazobactam + vancomycin reported a 2.5 increased relative risk $(P=.03)$ compared with pediatric patients receiving cefepime + vancomycin. ${ }^{33}$ Likewise, a multicenter study reported that vancomycin + piperacillin/tazobactam was associated with AKI in children (6 months-18 years of age) during the first week of hospitalization compared with children receiving vancomycin + a similar spectrum $\beta$-lactam agent. ${ }^{34}$ It is challenging to directly compare our results with other studies in pediatric patients because there are few studies investigating AKI risks for nephrotoxic drug combinations in infants, and no studies in adults or infants have directly compared nephrotoxicity for all of these drug combinations simultaneously.

There were no infants in our analysis who received chlorothiazide or spironolactone in combination with ibuprofen. Ibuprofen is most commonly administered to premature infants in the first few weeks after birth for the indication of patent ductus arteriosus. ${ }^{35}$ In contrast, infants may be more likely to receive diuretic therapy with chlorothiazide or spironolactone later in the hospital course to treat pulmonary sequelae of developing or established bronchopulmonary dysplasia. 
There may also be no infants receiving chlorothiazide + spironolactone or chlorothiazide + ibuprofen because we only considered the first course of combination therapy. Therefore, infants may have been excluded if they received other combination therapy, such as gentamicin + indomethacin or furosemide + gentamicin, before receiving these drug combinations.

As a retrospective study, our analysis was limited to medical and laboratory data reported in the medical chart, so we were unable to evaluate urine output or glomerular filtration rate. We excluded infants if they did not have $\geq 2$ serum creatinine values $(\mathrm{n}=15113)$ because we did not have sufficient data to conclude whether they experienced AKI during hospitalization. For stage 1 of the Kidney Disease: Improving Global Outcomes 2012 guidelines, the increase in serum creatinine values should be within 7 days of each another, however, we assessed the increase in serum creatinine during the exposure duration of nephrotoxic drug. ${ }^{13}$ This factor may slightly increase the association between AKI and nephrotoxic drugs, but should not affect the relative nephrotoxicity among drug combinations. Furthermore, our systematic approach of selecting nephrotoxic drug combinations was based upon the availability of good or excellent evidence for a nephrotoxic drug-drug interaction using Micromedex. Therefore, not every nephrotoxic drug combination encountered in the NICU was evaluated. We also did not account for drug dose or concentrations, but we did find that duration of drug combination therapy was associated with an increased risk of AKI. Future studies could be performed evaluating the association between dosing and nephrotoxic combination drug exposure with AKI in infants. In the Cox regression model, chlorothiazide + indomethacin was associated with an increased risk of AKI relative to gentamicin + indomethacin, but these findings may not be accurate given that there were only 6 infants receiving chlorothiazide + indomethacin. We also recognize that an assessment of AKI risks for nephrotoxic drug combinations relative to monotherapy of nephrotoxic drugs may be a more clinically useful analysis; however, we do not present such an analysis because of the inherent sampling bias in our dataset. Owing to clinician concerns for AKI in infants receiving nephrotoxic drug combinations, serum creatinine values were more frequently obtained in infants receiving nephrotoxic drug combinations. In contrast, serum creatinine values were likely only obtained in infants receiving monotherapy when there was a clinical suspicion of AKI. Thus, the incidence of AKI among infants receiving monotherapy was biased upward relative to infants receiving drug combinations (data not shown).

In this cohort of infants exposed to nephrotoxic drug combinations, the overall incidence of AKI was 17\%. Furosemide + tobramycin and vancomycin + piperacillin-tazobactam were associated with a decreased risk of AKI relative to gentamicin + indomethacin. The duration of combination therapy was associated with an increased risk of AKI regardless of the nephrotoxic drug administered. Therefore, administering combinations of nephrotoxic drugs for the shortest time possible or switching to a less nephrotoxic alternative agent may decrease the risk of AKI in infants.
Submitted for publication Mar 19, 2020; last revision received Aug 6, 2020; accepted Aug 13, 2020.

Reprint requests: Daniel Gonzalez, PharmD, PhD, UNC Eshelman School of Pharmacy, The University of North Carolina at Chapel Hill, 301 Pharmacy Lane, Campus Box \#7569, Chapel Hill, NC 27599-7569. E-mail: daniel.gonzalez@ unc.edu

\section{References}

1. Nada A, Bonachea EM, Askenazi DJ. Acute kidney injury in the fetus and neonate. Semin Fetal Neonatal Med 2017;22:90-7.

2. Jetton JG, Boohaker LJ, Sethi SK, Wazir S, Rohatgi S, Soranno DE, et al. Incidence and outcomes of neonatal acute kidney injury (AWAKEN): a multicentre, multinational, observational cohort study. Lancet Child Adolesc Health 2017;1:184-94.

3. Askenazi D, Abitbol C, Boohaker L, Griffin R, Raina R, Dower J, et al. Optimizing the AKI definition during first postnatal week using Assessment of Worldwide Acute Kidney Injury Epidemiology in Neonates (AWAKEN) cohort. Pediatr Res 2019;85:329-38.

4. Momtaz HE, Sabzehei MK, Rasuli B, Torabian S. The main etiologies of acute kidney injury in the newborns hospitalized in the neonatal intensive care unit. J Clin Neonatol 2014;3:99-102.

5. Selewski DT, Charlton JR, Jetton JG, Guillet R, Mhanna MJ, Askenazi DJ, et al. Neonatal acute kidney injury. Pediatrics 2015;136:e463-73.

6. Moffett BS, Goldstein SL. Acute kidney injury and increasing nephrotoxic-medication exposure in noncritically-ill children. Clin J Am Soc Nephrol 2011;6:856-63.

7. Rhone ET, Carmody JB, Swanson JR, Charlton JR. Nephrotoxic medication exposure in very low birth weight infants. J Matern Neonatal Med 2014;27:1485-90.

8. Slater MB, Gruneir A, Rochon PA, Howard AW, Koren G, Parshuram CS. Identifying high-risk medications associated with acute kidney injury in critically ill patients: a pharmacoepidemiologic evaluation. Pediatr Drugs 2017;19:59-67.

9. Hsieh EM, Hornik CP, Clark RH, Laughon MM, Benjamin DK Jr, Smith PB. Best Pharmaceuticals for Children Act-Pediatric Trials Network. Medication use in the neonatal intensive care unit. Am J Perinatol 2014;31:811-21.

10. Safina AI, Daminova MA, Abdullina GA. Acute kidney injury in neonatal intensive care: Medicines involved. Int J Risk Saf Med 2015;27:S9-10.

11. Constance JE, Balch AH, Stockmann C, Linakis MW, Korgenski EK, Roberts JK, et al. A propensity-matched cohort study of vancomycinassociated nephrotoxicity in neonates. Arch Dis Child Fetal Neonatal 2016;101:F236-43.

12. Zarfin Y, Koren G, Maresky D, Perlman M, MacLeod S. Possible indomethacin-aminoglycoside interaction in preterm infants. J Pediatr 1985;106:511-3.

13. Kidney International Supplements. Kidney Disease: Improving Global Outcomes (KDIGO). KDIGO clinical practice guideline for acute kidney injury. 2012. https://kdigo.org/guidelines/acute-kidney-injury/. Accessed January 24, 2019.

14. Gordjani N, Burghard R, Leititis JU, Brandis M. Serum creatinine and creatinine clearance in healthy neonates and prematures during the first 10 days of life. Eur J Pediatr 1988;148:143-5.

15. Miall LS, Henderson MJ, Turner AJ, Brownlee KG, Brocklebank JT, Newell SJ, et al. Plasma creatinine rises dramatically in the first 48 hours of life in preterm infants. Pediatrics 1999;104:e76.

16. Ito S. Pharmacokinetics 101. Paediatr Child Health 2011;16:535-6.

17. Pacifici GM. Clinical pharmacology of furosemide in neonates: a review. Pharmaceuticals (Basel) 2013;6:1094-129.

18. Charlton JR, Boohaker L, Askenazi D, Brophy PD, Fuloria M, Gien J, et al. Late onset neonatal acute kidney injury: results from the AWAKEN Study. Pediatr Res 2019;85:339-48.

19. El-Badawy AA, Makar S, Abdel-Razek AR, Abd Elaziz D. Incidence and risk factors of acute kidney injury among the critically ill neonates. Saudi J Kidney Dis Transplant 2015;26:549-55. 
20. Daga A, Dapaah-Siakwan F, Rajbhandari S, Arevalo C, Salvador A. Diagnosis and risk factors of acute kidney injury in very low birth weight infants. Pediatr Neonatol 2017;58:258-63.

21. Lee CC, Chan OW, Lai MY, Hsu KH, Wu TW, Lim WH, et al. Incidence and outcomes of acute kidney injury in extremely-low-birth-weight infants. PLoS One 2017;12:e0187764.

22. Andreoli SP. Acute renal failure in the newborn. Semin Perinatol 2004;28:112-23.

23. Bezerra CT, Vaz Cunha LC, Libório AB. Defining reduced urine output in neonatal ICU: importance for mortality and acute kidney injury classification. Nephrol Dial Transplant 2013;28:901-9.

24. Bolat F, Comert S, Bolat G, Kucuk O, Can E, Bulbul A, et al. Acute kidney injury in a single neonatal intensive care unit in Turkey. World J Pediatr 2013;9:323-9.

25. Allegaert K. The impact of ibuprofen or indomethacin on renal drug clearance in neonates. J Matern Neonatal Med 2009;22:88-91.

26. Gagliardi L. Possible indomethacin-aminoglycoside interaction in preterm infants. J Pediatr 1985;107:991-2.

27. Schentag JJ, Plaut ME, Cerra FB. Comparative nephrotoxicity of gentamicin and tobramycin: pharmacokinetic and clinical studies in $201 \mathrm{pa}$ tients. Antimicrob Agents Chemother 1981;19:859-66.

28. Smith CR, Lipsky JJ, Laskin OL, Hellmann DB, Mellits ED, Longstreth J, et al. Double-blind comparison of the nephrotoxicity and auditory toxicity of gentamicin and tobramycin. N Engl J Med 1980;302:1106-9.

29. Kumin GD. Clinical nephrotoxicity of tobramycin and gentamicin: a prospective study. JAMA 1980;244:1808-10.

30. Nahata MC. Lack of nephrotoxicity in pediatric patients receiving concurrent vancomycin and aminoglycoside therapy. Chemotherapy 1987;33:302-4.

31. Linder N, Edwards R, MeClead R, Mortensen ME, Walson P, Koren G. Safety of vancomycin with or without gentamicin in neonates. Neonatal Netw 1993;12:27-30.

32. Bhatt-Mehta V, Schumacher RE, Faix RG, Leady M, Brenner T. Lack of vancomycin-associated nephrotoxicity in newborn infants: a casecontrol study. Pediatrics 1999;103:e48.

33. Cook KM, Gillon J, Grisso AG, Banerjee R, Jimenez-Truque N, Phillips EJ, et al. Incidence of nephrotoxicity among pediatric patients receiving vancomycin with either piperacillin-tazobactam or cefepime: a cohort study. J Pediatric Infect Dis Soc 2018;8:221-7.

34. Downes KJ, Cowden C, Laskin BL, Huang YS, Gong W, Bryan M, et al. Association of acute kidney injury with concomitant vancomycin and piperacillin/tazobactam treatment among hospitalized children. JAMA Pediatr 2017;171:e173219.

35. Ohlsson A, Walia R, Shah SS. Ibuprofen for the treatment of patent ductus arteriosus in preterm or low birth weight (or both) infants. Cochrane Database Syst Rev 2018;9:CD003481. 